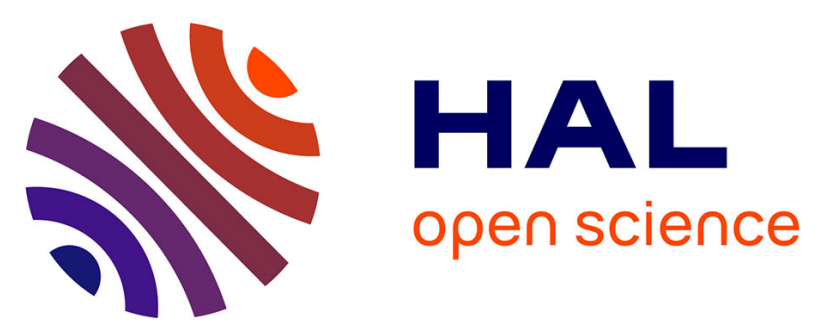

\title{
Dosimétrie in vivo par OSL, en ligne par fibre optique, pour l'assurance qualité des traitements par radiothérapie externe : le projet ANR-TECSAN Codofer
}

Sylvain Magne, Loïc de Carlan, A. Bridier, A. Isambert, Pierre Ferdinand, Régis Hugon, J. Guillon

\section{To cite this version:}

Sylvain Magne, Loïc de Carlan, A. Bridier, A. Isambert, Pierre Ferdinand, et al.. Dosimétrie in vivo par OSL, en ligne par fibre optique, pour l'assurance qualité des traitements par radiothérapie externe : le projet ANR-TECSAN Codofer. Innovation and Research in BioMedical engineering, 2010, 31 (2), pp.82 - 86. 10.1016/j.irbm.2010.02.002 . cea-01840732

\section{HAL Id: cea-01840732 \\ https://hal-cea.archives-ouvertes.fr/cea-01840732}

Submitted on 16 Jul 2018

HAL is a multi-disciplinary open access archive for the deposit and dissemination of scientific research documents, whether they are published or not. The documents may come from teaching and research institutions in France or abroad, or from public or private research centers.
L'archive ouverte pluridisciplinaire HAL, est destinée au dépôt et à la diffusion de documents scientifiques de niveau recherche, publiés ou non, émanant des établissements d'enseignement et de recherche français ou étrangers, des laboratoires publics ou privés. 


\title{
Dosimétrie in vivo par OSL, en ligne par fibre optique, pour l'assurance qualité des traitements par radiothérapie externe : Le projet ANR-TECSAN CODOFER
}

\section{Online Fibre Optic OSL in vivo Dosimetry for quality assurance of external beam radiation therapy treatments: The ANR-TECSAN CODOFER Project}

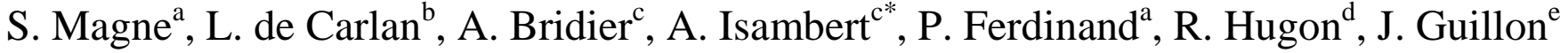 \\ ${ }^{a}$ CEA, LIST, Laboratoire de Mesures Optiques, F-91191 Gif-sur-Yvette, France \\ ${ }^{b}$ CEA, LIST, Laboratoire National Henri Becquerel, F-91191 Gif-sur-Yvette, France \\ ${ }^{c}$ Institut Gustave Roussy (Service de Physique) 39 rue Camille Desmoulins F-94805 Villejuif France \\ ${ }^{d}$ CEA, LIST, Département Capteur, Signal et Informations, F-91191 Gif-sur-Yvette, France \\ ${ }^{e}$ Société FIMEL, 18 rue Marie et Pierre Curie, F-92260 Fontenay-aux-roses \\ * Aurélie Isambert, actuellement à l'ASN, 6 place du Colonel Bourgoin 75012 Paris
}

\section{Résumé}

Le projet CODOFER (2007-2009), réalisé dans le cadre de l'appel ANR-TECSAN, a été initié par le CEA LIST, en partenariat avec l'IGR et la société FIMEL. Son objectif était de concevoir et valider aux plans métrologique et clinique des Capteurs à Fibres Optiques de type OSL dans le but de réaliser une Dosimétrie in vivo lors des traitements par radiothérapie externe avec des faisceaux d'électrons de haute énergie. Les résultats, combinés aux tests en environnement clinique réalisés par l'IGR dans le cadre du projet Européen MAESTRO, ont permis de démontrer le fort potentiel métrologique de la dosimétrie OSL/FO pour l'Assurance Qualité des traitements. Cependant, le calendrier réglementaire imposé par le gouvernement Français en 2007 a considérablement modifié l'état du marché français de sorte que l'application médicale du produit OSL/FO est actuellement ré-orientée vers d'autres modalités de traitement manquant de dosimètres adaptés (projet ANR-INTRADOSE (2009-2011)).

\begin{abstract}
The CODOFER Project (2007-2009), led under the ANR-TECSAN Call, was coordinated by CEA LIST, in partnership with IGR and the FIMEL company. The aim of the project was to design and test both metrologically and in clinical conditions OSL Optical Fiber Sensors dedicated to in vivo Dosimetry during external beam Radiation Therapy treatment with high-energy electrons. This study, combined with the results of clinical tests obtained within the European Project MAESTRO, has demonstrated the advantages of OSL/FO dosimetry for providing Quality Assurance of treatments. However, the French market for dosimetry has greatly changed as a result of the rules edicted by the French government in 2007. The OSL/FO product is now targeted for other treatment modalities lacking suitable dosimeters (ANR-INTRADOSE Project (2009-2011)).
\end{abstract}

Mots clés : Dosimétrie in vivo, Fibre Optique, OSL, Radiothérapie, Electrons

Keywords : In vivo Dosimetry, Optical Fibre, OSL, Radiotherapy, Electrons

\section{Contexte médical}

Actuellement, environ $60 \%$ des cas de cancers sont traités par radiothérapie (RT) seule ou en combinaison avec d'autres traitements (chimiothérapie, chirurgie). $\mathrm{Ce}$ traitement non mutilant requiert toutefois une grande précision afin de réduire les risques de cancers radio-induits et les effets secondaires indésirables.

La plupart des traitements par RT externe mettent en œuvre des faisceaux de photons et d'électrons produits par des accélérateurs linéaires (Linear Accelerators - LINACs). Les faisceaux de photons pénètrent profondément dans les tissus et interagissent donc de façon significative avec les tissus sains. Ainsi, une combinaison de faisceaux d'incidences angulaires, d'énergies et de géométries ajustées permet au médecin oncologue de délivrer la dose prescrite à la tumeur en épargnant les organes à risques (OAR) et les tissus sains environnants par une conformation optimisée de la distribution de dose. Les différentes étapes du traitement sont planifiées à l'aide d'un logiciel de planification de traitement (Treatment Planning System - TPS) à partir des données morphologiques du patient.

Le parcours des électrons dans le tissu est limité et dépendant de leur énergie, ce qui permet d'irradier une tumeur tout en préservant un OAR situé à proximité. En contrepartie, la surexposition de la peau est plus importante 
avec des électrons qu'avec des photons. Pour cette raison, les faisceaux d'électrons sont le plus souvent utilisés comme complément («boost») aux faisceaux de photons afin de préserver la peau située en amont de la tumeur et les OAR, en aval. Ils sont principalement utilisés pour traiter les chaînes mammaires internes (cancer du sein) et spinales (cancers Oto-Rhino-Laryngologiques), mais aussi pour les medulloblastomes (tumeurs cérébrales) et certains cas de cancers de la peau (Total Skin Electron Therapy - TSET).

\section{La Dosimétrie in vivo en Physique Médicale}

L'Assurance Qualité (AQ) des traitements par RT met en œuvre une Dosimétrie In Vivo (DIV) dans le but de vérifier que la dose délivrée correspond à la dose prescrite par le médecin oncologue. En RT externe, un dosimètre est placé dans le faisceau, en un point de référence sur la peau du patient. La dose mesurée est alors comparée à celle prévue par le TPS (au même point). En pratique, le dosimètre est inséré dans un capuchon d'équilibre électronique (build-up cap) qui le place à la position du maximum de dose $\left(\mathrm{d}_{\max }\right)$ et dont la géométrie peut aussi permettre d'assurer une réponse homogène en fonction de l'angle d'incidence du faisceau.

En pratique clinique, la DIV est le plus fréquemment appliquée au début et à chaque modification de traitement. En effet, l'insertion d'un dosimètre dans le faisceau modifie substantiellement la répartition des isodoses (atténuation par le détecteur) en comparaison de celles calculées par le TPS. De surcroît, il conduit à une surexposition de la peau puisque le maximum de dose apparait à cette interface. Enfin, la DIV prend du temps (placement, recueil \& analyse des données).

Avant 2007, la DIV faisait essentiellement partie d'une «bonne pratique médicale». Les récents accidents de surexposition de patients intervenus en France ont conduit les pouvoirs publics à modifier le Code de la Santé Publique dans le but d'améliorer la sécurité des patients [1]. L'application d'une DIV en routine constituera en 2011 l'un des critères d'agrément (opposables) retenus par l'Institut National du Cancer (INCa) et autorisant les établissements de santé à exercer une activité de soins en RT [2].

L'écart relatif entre les doses délivrée et prescrite ne doit pas dépasser $\pm 5 \%$ [3-4]. Les raisons d'un écart supérieur doivent être recherchées et des actions correctives, mises en application. Pour d'autres traitements impliquant des faisceaux «techniquement non mesurables » en l'état actuel de la technique [5] (Curiethérapie, Radiothérapie Conformationnelle à Modulation d'Intensité (RCMI), etc), la DIV n'est pas encore appliquée en routine.

Jusqu'à présent, les dosimètres les plus utilisés sont les diodes, les MOSFETS (Metal Oxyde Semiconductor Field Effect Transistor) et les TLD (thermoluminescence).

Les TLD à base de cristal LiF sont petits $\left(\mathrm{mm}^{3}\right)$, isotropes, équivalents-tissus, peu dépendants en température et ne présentent pas de dépendance envers le débit de dose. Le principal inconvénient des TLD réside dans leur lecture différée et le protocole d'évaluation et de régénération qui prend beaucoup de temps et nécessite une personne spécialisée pour ce travail.
Les MOSFETS sont également très petits et permettent d'effectuer des mesures de doses en ligne. Leur principal inconvénient tient à la saturation rapide de la tension de polarisation aux alentours de $250 \mathrm{~Gy}$. Aussi est-il nécessaire de les ré-étalonner et de les remplacer souvent, ce qui induit un surcoût important (consommable \& charge de travail).

Les diodes sont sensibles, peu coûteuses, abondamment utilisées car leur réponse est instantanée. Cependant, leur emploi met en œuvre un grand nombre de facteurs de correction liés à la dégradation de leur réponse avec la dose cumulée, au débit de dose, à la température, à l'énergie et l'incidence angulaire du rayonnement, ce qui a des répercussions en termes de charge de travail.

\section{La dosimétrie OSL/FO au CEA LIST}

Le CEA LIST bénéficie de ses travaux antérieurs en dosimétrie OSL par fibre optique (Optically Stimulated Luminescence/Fibre Optics - OSL/FO) [6]. Dans le cadre du Projet Européen MAESTRO (2004-2009) [7], le CEA LIST a mis au point une instrumentation OSL/FO multivoies de DIV pour l'AQ des traitements par RT externe, en ligne (i.e. capteur connecté en permanence). Celle-ci exploite la sensibilité élevée de cristaux d'alumine dosimétrique $\left(\mathrm{Al}_{2} \mathrm{O}_{3}: \mathrm{C}\right)$, optiquement transparents, de faible $\mathrm{Z}(\sim 11)$ et disponibles à un faible coût (USTU, Landauer).

Les Capteurs à Fibres Optiques (CFO) OSL testés dans cette étude incorporent une fibre cristalline TLD500 $(\mathrm{L}=1 \mathrm{~mm}$, $\varnothing=1 \mathrm{~mm}$ ) et sont constitués d'une tête de mesure solidaire d'un cordon à fibre $(12 \mathrm{~m})$ relié à l'instrumentation par un connecteur optique (fig. 2). Les CFO OSL sont pré-irradiés ( $200 \mathrm{~Gy}$ ) dans le but de stabiliser leur réponse en dose.

Sous irradiation, une partie des électrons libérés sont piégés dans le matériau (effet mémoire) tandis que d'autres se recombinent immédiatement en émettant une RadioLuminescence (RL), dont le suivi permet une mesure de dose en temps réel. Après irradiation, le CFO OSL est stimulé optiquement par un faisceau laser (@532 nm) transmis par la fibre optique et remis à zéro. La luminescence OSL (@ $410 \mathrm{~nm}$ ) consécutive à cette stimulation laser est collectée par cette fibre et transmise en retour à l'unité de mesure. Le signal OSL est proportionnel à la dose reçue entre deux stimulations successives. Celui-ci est alors corrigé du bruit de fond, intégré et multiplié par un coefficient d'étalonnage pour en déduire la dose.

Un commutateur optique permet de réaliser des mesures multivoies (15 voies), d'optimiser de facto le coût par point de mesure et de centraliser les données (fig. 1). Des tests en environnement clinique de l'instrumentation OSL/FO et des CFO OSL ont été effectués de manière autonome par le personnel de l'IGR avec des faisceaux de photons (projet MAESTRO) [10]. Un logiciel dédié (OSL EVOLUTION PRO), programmé par le CEA LIST selon les spécifications de l'IGR, réalise les étalonnages, les mesures de doses et comporte des auto-tests et les corrections en ligne.

Les performances de l'instrumentation OSL/FO du CEA LIST sont récapitulées dans le tableau Tab. 1 [8-10]. 


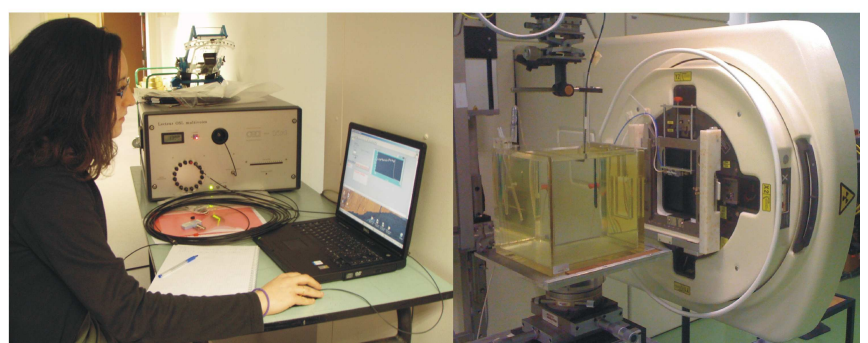

Fig. 1. Dosimètre OSL multivoies (gauche) développé par le CEA LIST dans le cadre du projet Européen MAESTRO et irradiation d'un CFO OSL en conditions de référence au CEA LIST LNHB (droite)

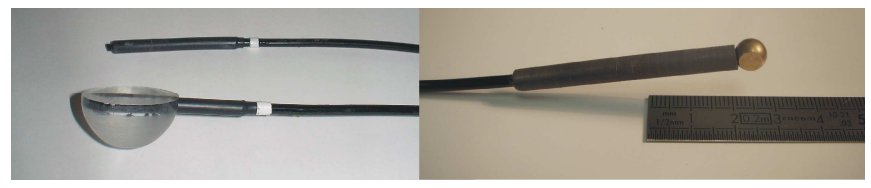

Fig. 2. CFO OSL solidarisés en extrémité de cordon optique (capteur nu, avec capuchons en PMMA (en bas, à gauche) et en laiton (à droite))

Tableau 1

Performances du système OSL/FO pour la DIV en RT externe.

\begin{tabular}{|c|c|}
\hline \multicolumn{2}{|l|}{ Paramètres } \\
\hline Mesures & en ligne (OSL) et temps réel (RL) \\
\hline Nombre de voies & 15 \\
\hline Durée de lecture (TLD500) & $\sim 40$ s par capteur \\
\hline Dépendance en température & entre $0,16 \% \cdot \mathrm{K}^{-1}$ et $0,3 \% \cdot \mathrm{K}^{-1}$ \\
\hline Perte d'information (fading) [8] & $-1 \%$ par décade en temps à $20^{\circ} \mathrm{C}$ \\
\hline Répétabilité (à 1 Gy) [8] & $\pm 1 \%$ \\
\hline Reproductibilité (à 1 Gy) [9] & $\pm 2 \%$ (dose cumulée de 64 kGy) \\
\hline Dépendance en énergie [8-10] & $\begin{array}{l} \pm 0,25 \% \text { sur }[9 \mathrm{MeV}, 18 \mathrm{MeV}] \text { et } \\
\pm 0,5 \% \text { sur }[6 \mathrm{MV}, 20 \mathrm{MV}]\end{array}$ \\
\hline Dépendance angulaire selon le & $\pm 1,5 \%$ sur $\left[-70^{\circ}, 70^{\circ}\right](\mathrm{PMMA})$ \\
\hline capuchon utilisé [10] & $\pm 2 \% \operatorname{sur}\left[-70^{\circ}, 70^{\circ}\right]$ (laiton) \\
\hline Dépendance au débit de dose & Aucune (capteur intégral) \\
\hline Stabilité sous irradiation [9] & Excellente \\
\hline Gamme de dose (TLD500) & [6 mGy, $6 \mathrm{~Gy}]$ \\
\hline Facteur de sortie [10] & Faible $( \pm 5 \%$ sur $[4 \mathrm{~cm}, 25 \mathrm{~cm}])$ \\
\hline Transparence au rayonnement & Excellente \\
\hline Compatibilité in vivo & $\begin{array}{l}\text { Bonne (capteur étanche, petit, inerte, } \\
\text { non-toxique, stérilisable) }\end{array}$ \\
\hline Immunité électromagnétique & Totale (lecteur hors salle de traitement) \\
\hline Coût du capteur & Modéré (estimé à 500 €) \\
\hline
\end{tabular}

\section{Le projet ANR-TECSAN CODOFER}

Le projet CODOFER (Capteurs OSL à fibres optiques pour la DOsimétrie des Faisceaux d'Electrons en Radiothérapie) regroupait le CEA LIST, l'IGR, centre référent en Radiophysique, et la société francilienne FIMEL, spécialisée en dosimétrie TL et analyse de faisceaux.

\subsection{Objectifs du projet CODOFER}

Les spécifications ont été fixées en adéquation avec les besoins des personnels de santé, dans le but d'assurer une DIV performante et simple à utiliser pour les traitements par
RT avec faisceaux d'électrons. La plupart des traitements par RT externe sont hypofractionnés ( 2 Gy par séance, 25 à 35 séances par traitement selon la localisation). Ainsi, une éventuelle erreur dans la délivrance d'une fraction de dose peut être corrigée à la séance suivante (la dose totale restant conforme à la prescription). Le consortium a donc choisi de mettre en œuvre la technique de dosimétrie OSL/FO.

Les objectifs du projet CODOFER étaient les suivants :

- Conception \& validation métrologique des CFO OSL pour les faisceaux d'électrons en RT externe,

- Test en environnement clinique à l'IGR,

- Transfert industriel auprès de la société FIMEL.

\subsection{Conception par simulation Monte-Carlo}

La géométrie des capuchons a été étudiée afin d'obtenir l'indépendance angulaire de la réponse en dose du capteur pour une mesure en surface du patient. Le code Monte Carlo MCNP5 (Los Alamos National Laboratory) a été utilisé. Un capuchon de géométrie optimale a été réalisé (fig. 2).

\subsection{Validations métrologiques}

Plusieurs validations métrologiques ont été menées en collaboration avec le LNHB-LMD (Laboratoire National Henri Becquerel), laboratoire primaire français en métrologie de la dose appartenant au CEA LIST.

\subsubsection{Réponse en dose et rendements en profondeur}

Les CFO OSL ont été testés selon le protocole TRS-398 [3] sous faisceaux d'électrons avec un LINAC Saturne 43 (énergies : 9, 12 et $18 \mathrm{MeV}$ ), dans la gamme [0, $3 \mathrm{~Gy}]$. Les CFO OSL ont été étalonnés en dose absorbée dans l'eau à la profondeur de référence (fig. 1) pour chaque énergie par comparaison avec une chambre d'ionisation (CI).
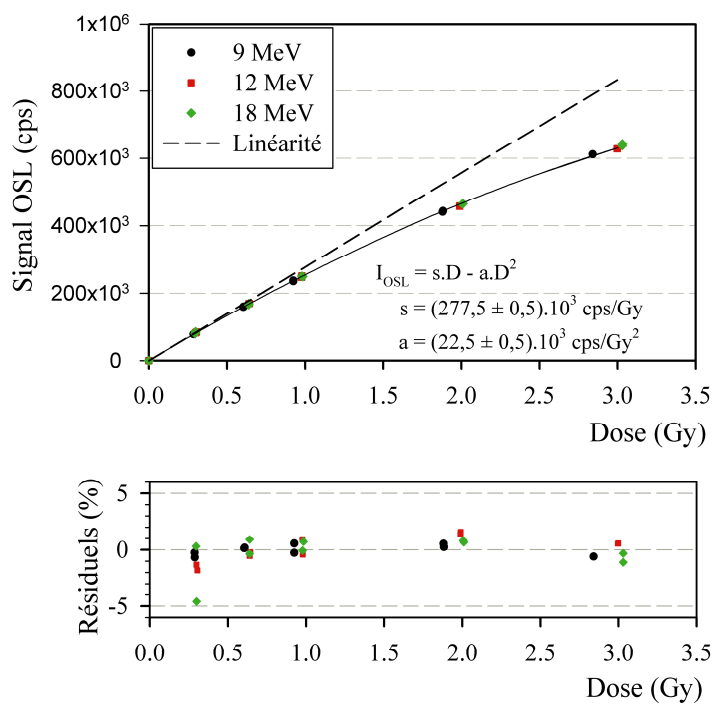

Fig. 3. Réponse en dose des capteurs OSL pour les 3 énergies de faisceaux d'électrons $(9,12$ et $18 \mathrm{MeV})$ et résiduels d'ajustement de second degré.

La réponse en dose (fig. 3) est non linéaire ( $\mathrm{I}_{\mathrm{OSL}}$ est le signal OSL intégré). L'affichage en dose par le logiciel OSL 
EVOLUTION PRO est linéarisé par la loi d'étalonnage jusqu'à une valeur maximale $\mathrm{s} / 2 \mathrm{a} \sim 6 \mathrm{~Gy}$.

La dépendance en énergie de la réponse en dose est faible $(\sim \pm 0,25 \%)$ en raison de la petite dimension du cristal $(\mathrm{mm})$ en comparaison du parcours des électrons (principe de Bragg-Gray). Une dépendance en énergie de l'ordre de $\pm 0,2 \%$ est calculée, conforme aux observations.

Le CFO OSL est ensuite déplacé à différentes profondeurs au sein du fantôme d'eau, le long de l'axe central du faisceau. La fig. 4 montre la bonne correspondance obtenue entre les doses mesurées par OSL et par CI $(\sim \pm 1 \%)$ [8].

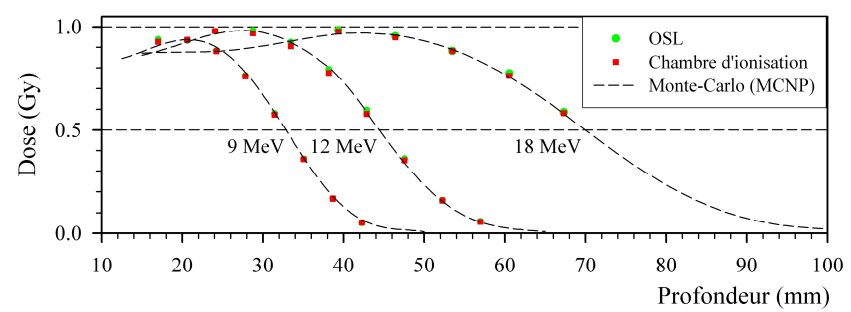

Fig. 4. Courbes de rendement en profondeur obtenues avec le capteur OSL et la CI et modélisation MCNP des distributions de dose (espace de phase exploitant un champ homogène et une distribution en énergie gaussienne).

\subsubsection{Indépendance angulaire}

Le CFO OSL monté dans son capuchon en PMMA est placé à la surface d'un fantôme d'eau. L'ensemble capteur \& fantôme est orienté par rapport à l'axe du faisceau de $0^{\circ}$ à $45^{\circ}$. La réponse en dose obtenue est stable selon une précision compatible avec la répétabilité de l'instrument [9].

\section{Bilan du projet \& Conclusions}

Les résultats obtenus grâce au projet CODOFER ont permis de combler une lacune de connaissances. Combinés à ceux obtenus dans le cadre du projet MAESTRO, ils ont permis de démontrer les avantages métrologiques d'une DIV par OSL/FO pour l'AQ des traitements par RT externe par faisceaux d'électrons.

En revanche, malgré ces résultats techniques prometteurs, l'opportunité d'un transfert industriel a disparu en cours de projet car le marché Français de la RT externe n'est plus porteur. En effet, le calendrier réglementaire imposé par le gouvernement Français en cours de projet (2007) a obligé les centres de soins à s'équiper dès 2008-2009 (principalement en diodes) et à former leurs équipes afin d'être en conformité avec cette nouvelle réglementation. A l'échéance médiane du projet, le consortium CODOFER a analysé le marché Français de la dosimétrie et en a conclu que le produit OSL/FO serait commercialisé trop tard, compte-tenu de l'effort restant à fournir pour le finaliser (optimisation du coût, intégration, marquage $\mathrm{CE}$, validations par les centres référents). Or son développement doit d'abord être amorti sur le marché Français avant d'envisager une exportation.

Dans ces conditions, la société FIMEL n'a pas souhaité prendre le risque d'industrialiser le lecteur OSL/FO et les CFO associés. Faute de démonstrateur, aucun test en environnement clinique n'a été réalisé en mode électron à l'IGR. L'IGR a cependant expertisé cette instrumentation innovante lors des tests cliniques (en mode photons) dans le cadre du projet MAESTRO [10].

Le CEA LIST poursuit actuellement des études de faisabilité pour des modalités de traitement pour lesquelles la DIV n'est pas encore appliquée en routine (car difficile à mettre en œuvre) et pour lesquelles la dosimétrie OSL/FO apporterait un avantage certain par rapport aux autres techniques disponibles. De plus, le mode temps réel est actuellement demandé par les physiciens médicaux pour tous les traitements. Ce mode serait à terme implémenté dans le logiciel EVOLUTION PRO. Ces études sont en cours dans le cadre du projet ANR-INTRADOSE (impliquant le CEA LIST et le Centre Léon Bérard, Lyon) dont l'objectif est d'établir la faisabilité d'une DIV intracavitaire pour les Curiethérapies et les RCMI. A terme, le CEA LIST souhaite industrialiser un dosimètre à fibre optique basé sur sa technologie propriétaire pour ces modalités de traitements particulières et ainsi contribuer à améliorer la sécurité des patients par une meilleure analyse des risques. Ce produit pourra être ultérieurement adapté pour la RT externe qui ne constitue actuellement qu'un marché de remplacement.

\section{Remerciements}

Cette étude a bénéficié des acquis du projet IP CE503564 MAESTRO (Methods \& Advanced Equipments for Simulation \& Treatments in Radio Oncology) avec l'aide de la Communauté Européenne. S. Magne remercie D. Chambellan pour le prêt d'un générateur X, A. Fallet \& G. Bouhot pour les calculs MCNP, C. LeBret \& E. Spasic pour leurs contributions aux validations métrologiques et cliniques, $P$. Escobar pour la programmation.

\section{Pour en savoir plus}

[1] Décrets n ${ }^{\circ} 2007-388 \& 389$ du 21/03/2007.

[2] Circulaire DHOS/E4 n²007-230 du 11/06/2007.

[3] IAEA, TRS 398, Absorbed dose determination in external beam radiotherapy: An international Code of Practice for dosimetry based on standards of absorbed dose to water, 2000, Vienna, Austria.

[4] Huyskens, Bogaerts R., Verstraete J., Lööf M., Nyström H., Fiorino C. et al., Practical guidelines for the implementation of in vivo dosimetry with diodes in external radiotherapy with photon beams (entrance dose) ESTRO Booklet n5-2001.

[5] Beauvais-March H., Estivalet S., Goubard O., Guide pour la pratique quotidienne de la DIV en radiothérapie externe, Rapport rédigé sous l'égide de l'INCa avec la collaboration de la SFPM et de l'ASN, Oct. 2008

[6] Ranchoux G., Magne S., Bouvet J.P., Ferdinand P., Fiber Remote Optoelectronic gamma dosimetry based on OSL of $\mathrm{Al}_{2} \mathrm{O}_{3}: \mathrm{C}$, Rad. Prot. Dos. $2002 ; 100,1-4$, , 255-260.

[7] Barthe J., Hugon R., Nicolai J. Ph., MAESTRO: Methods and Advanced Equipment for Simulation and Treatments in Radio-Oncology, Nucl. Instr. In Phys. Res. A. 2007 ; 583, 1-8.

[8] Magne S., Auger L., Bordy J. M., de Carlan L., Isambert A., Bridier A. et al.., Multichannel dosemeter and $\mathrm{Al}_{2} \mathrm{O}_{3}: \mathrm{C}$ OSL fibre sensors for use in radiation therapy - Evaluation with electron beams, Radiat. Prot. Dosim.. $2008 ; 131,1,93-99$.

[9] Magne S., De Carlan L., Sorel S., Isambert A., Bridier A., Ferdinand P., Barthe J., Multichannel OSL dosimetry for dose verification in radiotherapy, $12^{\text {th }}$ International Congress IRPA, Buenos Aires, 19-24 Oct. 2008.

[10] Magne S., de Carlan L., Bordy J.M., Isambert A., Bridier A., Ferdinand P., Multichannel Dosimeter and $\alpha-\mathrm{Al}_{2} \mathrm{O}_{3}$ :C OSL fibre sensors for use in RT therapy - Evaluation with photon beams, Submitted for publication in IEEE Trans. Nucl. Sci. 